\title{
Qualidade de vida relacionada ao ambiente ocupacional dos agentes penitenciários do estado do maranhão
}

RESUMO | Objetivo: identificar o nível de qualidade de vida segundo o ambiente ocupacional das Unidades Prisionais. Método: Pesquisa transversal, qualitativa, não-probabilista, com 104 participantes, no Estado do Maranhão, entre junho de 2019 a julho de 2020. Resultados: A maioria dos participantes são do sexo masculino $84 \%$, onde $63 \%$, possuem ensino superior. 0 domínio qualidade de vida apresentou melhor média 54,7. Enquanto o domínio ambiente ocupacional apresentou a pior média 35,8. Quando realizada a comparação entre quais gêneros apresentaram os melhores escores nos 5 domínios, houve igualdades entre os dois gêneros. Conclusão: Os resultados demonstram que os agentes penitenciários possuem bom nível de qualidade de vida em todos os domínios do QVS-80. Porém, o domínio ambiente ocupacional, apresentou os piores escores, demonstrando ser um ponto que necessita de melhorias, uma vez que estes servidores passam boa parte do tempo de suas vidas nestes locais.

Palavras-chaves: Qualidade de Vida; Penitenciária; Saúde Pública.

ABSTRACT | Objective: to identify the level of quality of life according to the occupational environment of the Prison Units. Method: Cross-sectional, qualitative, non-probabilistic research, with 104 participants, in the State of Maranhão, between June 2019 and July 2020. Results: Most participants are 84\% male, where $63 \%$ have higher education. The quality of life domain had a better average of 54,7 . While the occupational environment domain had the worst average of 35,8 . When comparing the genders with the best scores in the 5 domains, there was equality between the two genders. Conclusion: The results demonstrate that prison officers have a good level of quality of life in all domains of the QVS-80. However, the occupational environment domain had the worst scores, proving to be a point that needs improvement, since these servers spend a good part of their lives in these places.

Keywords: Quality of Life; Penitentiary; Public health.

RESUMEN | Objetivo: Identificar el nível de calidad de vida acorde al ambiente ocupacional de las unidades penitenciarias. Método: Investigación transversal, cualitativa, no probabilística, com 104 participantes, em el estado de Maranhão, entre junio de 2019 y julio de 2020. Resultados: La mayoría de los participantes son $84 \%$ hombres, donde el $63 \%$ tiene educación superior. El domínio de la calidad de vida tuvo um mejor promedio de 54,7. Mientras que el domínio del entorno ocupacional tuvo el pero promedio de 35,8. Al comparar qué géneros obtuvieron las mejores puntuaciones em los 5 dominios, hubo igualdad entre los dos géneros. Conclusión: Los resultados demuestran que los funcionários de prisiones tienen um buen nível de calidad de vida en todos los domínios del QVS-80. Sin embargo, el domínio de ambiente ocupacional uvo las peores puntuaciones, demostrando ser um punto que necesita mejora, ya que estos servidores pasan buena parte de sua vida em estos lugares.

Palabras claves: Calidad de vida; Penitenciario; Salud Pública.

\section{Felipe Santana e Silva}

Mestrando em Biodiversidade Ambiente Saúde. Universidade Estadual do Maranhão - UEMA.

ORCID: 0000-0002-5919-8213

\section{Joseneide Teixeira Câmara}

Doutora em Medicina Tropical. Universidade Estadual do Maranhão - UEMA.

ORCID: 0000-0002-8312-1697

\section{Raimundo Nonato Silva Gomes}

Doutor em Engenharia Biomédica. Universidade Federal do Rio de Janeiro - RJ. ORCID: 0000-0002-8739-2734

Recebido em: 20/05/2021

Aprovado em: 08/06/2021

INTRODUÇÃO

$\Lambda$ qualidade de vida é uma entidade multidimensional que inclui repercussões nas mais variadas dimensões físicas, psicológicas, sociais e ambientais, não se atentando assim, apenas para a ausências de enfermidades. Portanto, instrumentos para a avaliação desta entidade são necessários como é o caso do Questionário de Avaliação da Qualidade de Vida e da Saúde (QVS-80), que é um instrumento que busca avaliar a Qualidade de vida e a saúde nos trabalhadores nos mais diversos ambientes ocupacionais. ${ }^{1}$

Considerado um dos maiores estudiosos, sobre a temática Qualidade de Vida no Trabalho (QVT), Walton, define a mes- ma com sendo calcada em humanização do trabalho e responsabilidade social da instituição, envolvendo o entendimento de necessidades e aspirações do indivíduo, por meio da reestruturação do desenho de cargos e nova formas de organizar o trabalho, aliado a formação de equipes de trabalho com maior poder de autonomia e melhoria do meio organizacional. Desta forma, salienta-se a dificuldade dos trabalhadores em conciliar a QV e as atividades laborais, principalmente quando o cenário laboral são as penitenciárias brasileiras. $^{2}$

O agente penitenciário é o profissional que realiza um serviço público de alto risco através do tratamento penal, da custodia e da vigilância da pessoa presa no sistema prisional. Haja vista, que exis- 
te um perfil adequado para exercer essa profissão como ter aptidão, responsabilidade, iniciativa, honestidade, lealdade, disciplina, equilíbrio emocional, liderança, empatia, entre outros. ${ }^{3}$

Embora, a profissão de Agentes Penitenciários esteja diretamente ligada a preservação da ordem pública, o estigma envolvido à instituição de trabalho, além do alto nível de estresse a que estão submetidos, prejudica a interação social destes profissionais, isso acarreta no desenvolvimento de doenças. Por ser considerada a segunda profissão mais perigosa do mundo, uma vez que apresenta, paralelamente, risco de periculosidade e insalubridade, os AP, vivenciam elevadas cargas de estresse e risco, assim esses fatores ocasionam o comprometimento físico e mental destes indivíduos. ${ }^{4}$

Entende-se que quando os profissionais estão expostos a condições mínimas de trabalho para Ihe dá com pessoas desprovidas de liberdade, onde estes, tem suas necessidades básicas reduzidas, os mesmos encontram-se mais vulneráveis a repercussões insalubres na sua saúde física e mental. O que leva consequentemente a um agravo na sua qualidade de vida e profissional. ${ }^{5}$

Assim sendo, este trabalho tem como objetivou identificar o nível de qualidade de vida segundo o ambiente ocupacional das Unidades Prisional do estado do Maranhão, segundo a visão dos Agentes Penitenciários, que atuam nestes locais.

\section{MÉTODOS}

Foi realizada uma pesquisa transversal de abordagem qualitativa, uma vez que buscou-se a familiarização do pesquisador com o objeto de interesse e aplicação do conhecimento. Utilizou-se uma abordagem quantitativa, em relação a classificação.

O estudo ocorreu com um total de 104 agentes penitenciários do estado do Maranhão, estado este localizado na região Nordeste do Brasil. A amostra foi definia mediante elementos não probabi- lísticos, a partir de critérios de inclusão e exclusão predefinidos. Os critérios de inclusão foram ser Agente Penitenciário e trabalhar diretamente no ambiente prisional. Para exclusão utilizou-se os seguintes critérios: os AGEPENS que não possuem e-mail, para o envio online do questionário, os que por algum motivo estejam incapacitados para responder a pesquisa ou que não concordem em participar da mesma, não assinando o Termo de Consentimento Livre e Esclarecido (TCLE).

A coleta de dados ocorreu entre os meses de Junho de 2019, a julho de 2020, após aprovação no Comitê de Ética em Pesquisa (CEP), da Universidade Estadual do Maranhão (UEMA), conforme o CAAE: 21016619.0.0000.5554/n $\mathrm{n}^{\circ}$ do parecer: 3.628.072, conforme deliberado pela Conselho Nacional de Saúde, na resolução CNS 466/12. Para a coleta dos dados utilizou-se a aplicação de um questionário denominado Questionário de Avaliação da Qualidade de Vida e da Saúde (QVS-80). O questionário foi enviado aos pesquisados por um link através do e-mail destes, através de uma plataforma online denominada Google Forms.

Este questionário foi aplicado de forma online, através de uma plataforma, denominada Google Forms. Onde, o QVS80, foi disponibilizado através de um link, e consequentemente enviado através do WhatsApp, para o todos os diretores das Unidades Prisionais do Maranhão, que enviaram para os grupos de agentes de cada unidade onde estes atuam.

Para caracterizar assim o ambiente, no qual estes atuam, utilizou-se o Questionário de Avaliação da Qualidade de Vida e da Saúde (QVS-80). Este instrumento é composto por oitenta questões, das quais 67 foram estruturadas na Escala de Likert e dividido em quatro domínios que são: Domínio da saúde (Saúde); Domínio da atividade física (AF), Domínio do ambiente ocupacional (AO) e Domínio da percepção da Qualidade de Vida (QV).

Os dados foram tabulados e analisados utilizando o Microsoft Excel. Onde utilizou-se análises descritivas de tendên- cia central, como média e mediana, além de medidas de dispersão como desvio padrão (DP), variância, valores máximos, mínimo, frequência e porcentagem. Só após essas analises procedeu-se a discussão dos achados com base na literatura produzida sobre o tema.

Para conferir confiabilidade ao estudo, foi realizada uma análise dos dados, através do Alfa de Croncbach. Além disso, visando equalizar as etapas de calibração dos avaliadores do estudo e padronização do processo de avaliação da concordância, foi realizado o cálculo de Coeficiente de Correlação Intraclasse $(\mathrm{CCl})$.

Uma vez que cada questão do QVS80 está em escala Likert de 5 pontos, sendo que os números se dão em ordem crescente. Após o preenchimento do questionário, os valores foram somados e normatizados para uma escala de 0-100 pontos para cada domínio. Onde a pontuação deste instrumento classificou o instrumento em: excelente ( $\geq 75$ pontos); bom ( $\geq 50$ e $<75$ pontos); regular ( $\geq 25$ e $<50$ pontos); ruim ( $\leq 25$ pontos) ${ }^{6}$

\section{RESULTADOS}

Ao realizar-se a descrição dos itens, os pesquisadores obtiveram, um total de 104 participantes, sendo que deste número $101(97,1 \%)$, foram validos, e que apenas $3(2,9 \%)$, foram excluídos. O que demonstra um excelente resultado de confiabilidade, uma vez que obteve-se um índice de validade acima de 95\%. Levando-se em consideração o Alfa de Cronbach com base nos itens padronizados, o resultado chega à 0,903 , em um total de 80 itens avaliados, ou seja, as 80 questões do QVS-80. Considerando-se que um índice de 0,81 a 1,0 é quase perfeito, entende-se que o questionário teve um bom índice de confiabilidade. Além disso, visando equalizar as etapas de calibração dos avaliadores do estudo e padronização do processo avaliação da concordância, calculou-se o coeficiente de correlação intraclasse, que teve um resultado excelente $(0,874)$. 
Tabela 1 - Caracterização dos Agentes Penitenciários participantes do estudo, segundo o sexo, estado civil e grau de instrução. Caxias, MA, Brasil, 2020.

\section{Caracterização}

\section{Maranhão}

$\mathrm{N}=104$

Sexo

\begin{tabular}{|lcc|}
\hline Masculino & 87 & 84 \\
\hline Feminino & 17 & 16 \\
\hline Estado Civil & & 59 \\
\hline Solteiro (a) & 60 & 39 \\
\hline União Estável & 40 & 3 \\
\hline Divorciado (a) & 2 & 3 \\
\hline Viúvo & 2 & \\
\hline Grau de Instrução & & 5 \\
\hline Primeiro grau & 4 & 2 \\
\hline Segundo grau incompleto & 1 & 9 \\
\hline Segundo grau & 8 & 63 \\
\hline Superior & 65 & 25 \\
\hline Pós-graduação & 26 & \\
\hline
\end{tabular}

Fonte: Autores, 2021.

Tabela 02 - Descrição da qualidade de Vida (QVS-80), dos agentes penitenciários, de acordo com a média, desvio padrão, mínimo e máximo. Caxias, MA, Brasil, 2020.

\begin{tabular}{|lccccc|}
\hline Domínios (QVS-80) & $\begin{array}{c}\text { Maranhão } \\
\text { Média }\end{array}$ & Desvio Padrão & Mínimo & Máximo & Mediana \\
\hline Saúde & 36,4 & 7,9 & 19 & 60 & 36 \\
\hline Atividade Física & 45,6 & 9,1 & 28 & 67 & 43,5 \\
\hline Ambiente Ocupacional & 35,8 & 7,4 & 13 & 67 & 36 \\
\hline Qualidade de Vida & 54,7 & 13,9 & 28 & 96 & 53,5 \\
\hline
\end{tabular}

Fonte: Autores, 2021. Legenda: O valor zero corresponde ao resultado mais negativo (pior qualidade de vida) e 100 corresponde ao resultado mais positivo (melhor qualidade de vida).

Tabela 03 - Distribuição da média dos escores de qualidade de vida do (QVS-80) dos agentes penitenciários do estado do Maranhão, segundo o sexo e o estado civil. Caxias, MA, Brasil, 2020.

\begin{tabular}{lccccc|}
\multicolumn{1}{l}{ Variáveis } & $\begin{array}{l}\text { Domínios } \\
\text { Saúde }\end{array}$ & AF & AO & QV & Qualidade de vida Geral \\
\hline Sexo & & & & & \\
\hline Masculino & 36,3 & 45,8 & 35,6 & 54,2 & 64 \\
\hline Feminino & 37,2 & 44,7 & 34 & 56,9 & 64 \\
\hline Estado Civil & & & & & \\
\hline Solteiro & 36,3 & 44,7 & 35,2 & 53,3 & 68 \\
\hline União Estável & 36,2 & 47,3 & 36,4 & 55,5 & 65 \\
\hline Divorciado(a) & 33,5 & 43,5 & 37 & 54 & 62 \\
\hline
\end{tabular}

Os dados da tabela 01, demonstram que a maioria dos participantes são do sexo masculino (84\%), isso se dá pelo fato de que a maioria dos agentes penitenciários pertencerem a esse sexo. Em relação ao estado civil grande parte dos AP são solteiros, este número correspondeu a $59 \%$.

Quando perguntados qual o grau de instrução dos mesmos $63 \%$, destes tem ensino superior, e apenas $5 \%$, tem primeiro grau, estes participantes que tem primeiro grau, são também os agentes com maior tempo de serviço, uma vez que os primeiros concursos para essa categoria não exigiam ensino superior. Percebe-se ainda que $25 \%$ dos integrantes do estudo tem uma Pós-graduação, que revela que apesar de estarem em um emprego estável, estes ainda se preocupam em qualificar-se.

Na segunda tabela, foram avaliados os quatro domínios do questionário em cinco variáveis diferentes que foram: média, desvio padrão, mínimo, máximo e mediana. Diante dos dados, percebe-se que os participantes obtiveram melhores números no domínio qualidade de vida, que é o principal domínio avaliado pelo QVS-80, onde obteve-se: média de $(54,7)$; desvio padrão de (13,9); mínimo de (28); máximo de (96) e mediana de $(53,5)$.

Já o domínio ambiente ocupacional, dentre todos os outros apresentou as variáveis com menores valores que foram sucessivamente: média $(35,8)$; desvio padrão $(7,4)$; mínimo (28); máximo $(67)$ e mediana (36).

$\mathrm{Na}$ tabela acima, foi realizado um levantamento onde buscou-se avaliar qual sexo e qual estado civil, apresentou o melhor escore de qualidade de vida nos quatro domínios do QVS-80. Os dados revelam que na qualidade de vida geral que é a soma dos quatro domínios tanto o sexo masculino, quanto o sexo feminino, apresentaram a mesma pontuação, ou seja, 64 pontos o que na escala de Likcter, significa um bom escore de qualidade de vida.

Quando analisado o estado civil, os solteiros demonstram ter melhor QV de vida com 68 pontos. Os divorciados (a), 


\section{Viúvo (a)} 46 47,5

39,5 87 66

Fonte: Autores, 2021. Legenda: O valor zero corresponde ao resultado mais negativo (pior qualidade de vida) e 100 corresponde ao resultado mais positivo (melhor qualidade de vida). Atividade Física (AF); Ambiente Ocupacional (AO); Qualidade de Vida (QV).

por sua vez demonstraram uma pior escore, porém, ainda considerado bom, levando em consideração a escala e Likcter.

\section{DISCUSSÃO}

Em relação aos dados da tabela 01 , referente ao gênero, esses números encontram semelhança com um estudo realizado em João Pessoa, estado da Paraíba, onde o número de Agentes Penitenciários do sexo masculino é maior que do sexo feminino. $\quad \mathrm{Na}$ pesquisa em questão realizando no ano de 2010, o número de agentes do sexo masculino foi de $80,8 \%$. Estes números se dão por dois motivos, o fato de ser um trabalho voltado a população masculina, uma vez que as revistas intimas dos internos incluem a retirada da vestimenta dos mesmos e principalmente na limitação em alguns concursos, onde o edital traz o número limite de mulheres para ingresso em determinados certames. ${ }^{7}$

Outra semelhança de dá em relação ao estado civil, onde na mesma pesquisa citada no parágrafo anterior $48,1 \%$, declaram ser solteiros e apenas 9,6\% divorciados. Números similares ao deste estudo, onde 59\% são solteiros e apenas 3\% divorciados. Estes dados são de grande relevância, uma vez que o estado civil, influi diretamente no estado de qualidade de vida, o que acarreta diretamente na forma de trabalho dos profissionais, principalmente em se tratando da segunda profissão mais perigosa do mundo. ${ }^{8}$

$\mathrm{Na}$ tabela 02 , os números mostram que os participantes tiveram bons escores em todos os domínios. Porém, no domínio ambiente ocupacional, esses números foram menores quando comparados aos demais. Isso vai de encontro com o que dizem os autores Luxen et al., que em sua pesquisa, relatam que os agentes penitenciários, atuam em um ambiente, vulnerável a situações de risco a saúde mental pelo desenvolvimento dos mais variados sintomas, e que isso se dá devido à natureza, que se apresentam, ou seja, um ambiente de sofrimento humano, hostilidades, conflitos interpessoais e os impactos dos limites institucionais para o exercício profissional. ${ }^{5}$

Perceptível aos achados encontrados no parágrafo anterior, em um estudo que analisou os fatores intervenientes na qualidade de vida de trabalhadores de enfermagem em jornada noturna. Os autores do estudo, revelam que o tempo de trabalho na instituição e o tempo de atuação no período noturno, influenciam negativamente na QV, dos trabalhadores. Isso assemelhasse as longas jornadas dos Agentes Penitenciários, onde estes trabalham por longos períodos noturno, as vezes sem descanso, por conta do pouco efeito e com medo de algum motim nas unidades prisionais. ${ }^{9}$

Já o domínio ambiente ocupacional, apresentou os menores escores uma vez que este é composto por inúmeras facetas que abordam a segurança física, ambiente do lar, recursos financeiros além de cuidados sociais e com a saúde. Uma vez que existe um estigma associado à instituição de trabalho e o alto nível de estresse a que estes trabalhadores estão submetidos acarreta o desenvolvimento de doenças. ${ }^{10-11}$

Observa-se que na tabela 03, na qualidade de vida geral ambos os gêneros demonstraram tem uma boa qualidade de vida, uma vez que os dois apresentaram 64 pontos, o que na escala de Likert é considerado bom. Já quando, levou-se em consideração, o estado civil dos participantes, o resultado também foi bom, uma vez que todos apresentaram-se acima de 62 pontos o que também é considerado bom, levando-se em consideração a mesma escala. Resultado semelhante, foi encontrado em um estudo realizado na cidade de Cajazeiras-PA, no qual os autores obtiveram um escore de
71,07 pontos, utilizando o questionário de avaliação de vida WHOQOL-Bref. Onde, os dados demonstraram um bom nível de QV, estes atribuíram o resultado como satisfatório, quando se relaciona a importância atribuída ao trabalho dos agentes penitenciários, o que indica satisfação no trabalho, ambiente ocupacional este avaliado pelo estudo. ${ }^{12}$

Entende-se que apesar do achado geral ser considerado bom em todos os domínios avaliados pelo questionário, ressalta-se a importância de adotar-se um programa de QV no trabalho, voltado exclusivamente para os agentes penitenciários, no qual este tenha como propósito melhorar a satisfação e o bem-estar destes servidores. Estás medidas, irão melhorar a produtividade e o desempenho, visto que, está intimamente ligada ao nível de satisfação no ambiente de trabalho, desta forma alcançando mais objetivos organizacionais, que se condicionam aos interesses individuais, principalmente quando leva-se em consideração o mercado competitivo que tem-se hoje. ${ }^{7}$

O ambiente ocupacional é psicologicamente exigente, uma vez que as condições de trabalho nas unidades prisionais são precárias em virtude da superlotação, do ambiente insalubre e falta de estrutura. Somando-se a isso a falta de matérias de proteção, algemas, fardamentos, armamentos e efetivo suficiente. Soma-se a isso a negligencia dos órgãos fiscais e da própria sociedade para oferecer melhores condições trabalhistas aos agentes penitenciários. ${ }^{13-14}$

Para maior confiabilidade do estudo além de calcular o Alfa de Cronbach, os autores equalizaram as etapas do estudo e padronização da concordância, através do $\mathrm{CCl}$, que apresentou um resultado excelente. Este coeficiente é uma das ferramentas mais utilizadas para mensuração da confiabilidade de medidas, e pode ser utilizado para mensuração da homogeneidade de duas ou mais medidas, como no caso desta pesquisa, além disso é interpretado como a medida da proporção da variabilidade total atribuída ao objeto 
medido, uma vez que baseia-se em uma razão de variâncias, onde nem sempre os métodos clássicos são capazes de estimar estes componentes. ${ }^{15}$

\section{CONCLUSÃO}

Os achados desta pesquisa realizada com os agentes penitenciários do estado do maranhão, indicou-se como positiva em todos os domínios que envolvem o questionário aplicado na mesma. Deste modo, destaca-se que o objetivo geral deste estudo foi alcançado. Uma vez que a mesma buscou identificar o nível de qualidade de vida destes trabalhadores relacionada o ambiente ocupacional dos mesmos.

Para conferir maior confiabilidade a esta pesquisa, os autores se utilizaram de dois métodos que foram: o Alfa de Cronbach que inferiu que o instrumento utilizado na pesquisa se apresentou como relevante e por fim o Coeficiente de Correlação Interclasse, que visou a padronização de todo o processo e avaliação da concordância do mesmo, garantido assim excelente grau de confiabilidade ao estudo.
Referindo-se a qualidade de vida dos AGEPENS, percebe-se que estes apresentam um bom nível de qualidade de vida, mesmo quando correlacionasse os domínios contidos no questionário, com

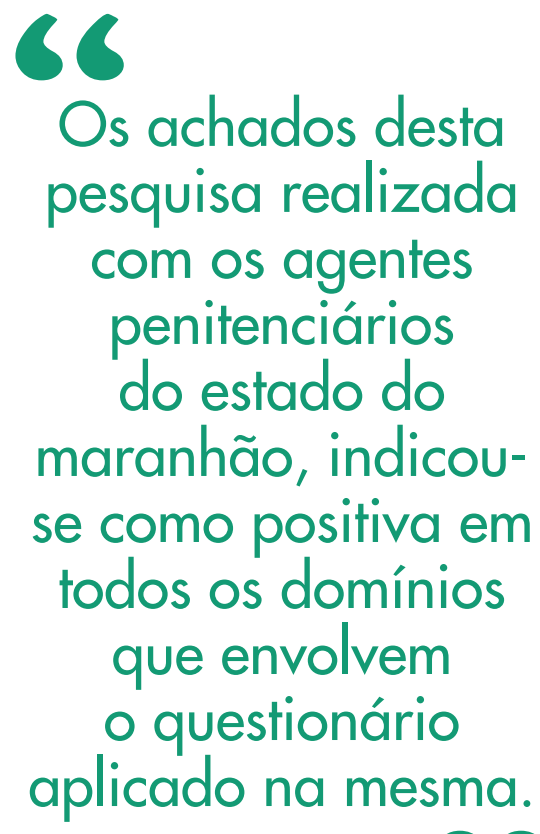

98 o gênero, estado civil ou grau de escolaridade. Onde, todo estes apresentam bons escores, segundo a escala de Likert, utilizada nesta pesquisa.

Porém, como era de espera-se o domínio ambiente ocupacional, mesmo apresentando-se como bom, este apresentou os piores escores. O que sugere que intervenções sejam feitas, afim de promover um ambiente ocupacional, que tenha segurança e assim possa promover a saúde desta classe, com vistas melhorar ainda mais as condições de vida, de trabalho e consequentemente a qualidade de vida dos agentes penitenciários.

Os resultados deste estudo podem contribuir com a gestão do sistema penitenciário, tendo em vista o reconhecimento dos escores com menor pontuação, ao fornecer embasamento para gestão adequá-las, com o intuito de garantir uma melhor infraestrutura de trabalho, desse modo, resultados mais eficientes, no que diz a QV de vida dos AGEPENS, esperam-se ser alcançados a partir da melhoria do ambiente ocupacional, no qual estes, encontram-se inseridos.

\section{Referências}

1. Jesus NM, Souza GF, Mendes-Rodrigues C, Almeida Neto OP, Rodrigues DDM, Cunha CM. Qualidade de vida de indivíduos com doença renal crônica em tratamento dialítico. J. Bras. Nefrol. 2021; 41 (3): 364-74.

2. Barbosa ML, Menezes TN, Santos SR, Olinda RA, Costa GMC. Qualidade de vida no trabalho dos profissionais de saúde no sistema prisional. Ciência \& Saúde Coletiva, 2018; 23(4):1293-02.

3. Lins ALV. Uma revisão integrativa sobre a saúde mental dos agentes de segurança penitenciária [dissertação]. Campina Grande (JP): Universidade Estadual da Paraíba; 2019.

4. Ramos FMC, Morais HCC, Campos RKG, Mendes IC. Qualidade de vida no trabalho do agente penitenciário cearense. Revista de Enfermagem da UFPI. 2021; 10(1):1-7.

5. Lauxen IAG, Borges RSS, Silva MB. A gestão penitenciária na qualidade de vida profissional do servidor penitenciário. Saúde em Redes, 2017; 3(3):256263.

6. Grande AJ, Silva V, Manzatto L, Rocha TB, Martins CG, Vilela Junior GB. Comparação de intervenções de promoção à saúde do trabalhador: ensaio clínico controlado randomizado por cluster. Rev Bras Cineantropom Desempenho Hum [Internet]. 2013; 15(1): 27-37.

7. Oliveira JS, Walter F, Pinho MAB, Amorim TNGF. Qualidade de Vida no Trabalho: um estudo sobre a avaliação por Agentes de Segurança Penitenciária. RaUnP, [Internet]. 2013; 5(2):63-77.

8. Hoertel R. Notícias Saúde e Ciência. Organização registra 160 milhões de casos de doenças relacionadas ao trabalho por ano. 2013 [cited $2021 \mathrm{Abr}$ 15]. Available from: http://extra.globo.com/noticias/saude-e-ciencia/organizacao-registra-160-milhoes-de-casos-de-doencas-relacionadas-ao-trabalho-por-ano-8246681.html.

9. Nougueira da Silva PLN, Pereura IL, Galvão APC, Figueiredo ML, Alves CR, Martins AG. Fatores intervenientes na qualidade de vida de trabalhadores de enfermagem em jornada noturna. Rev.Nursing [Internet]. 2021; 24(272): 5172-5184.

10. Bonez A, Moro ED, Sehnem SB. Saúde mental de agentes penitenciários de um presídio catarinense. Psicol. Argum [Internet]. 2013; 31(74): 507-17. 11. Rudnicki D, Schäfer $G$, Silva JC. As máculas da prisão: estigma e discriminação das agentes penitenciárias. Revista Direito GV [Internet]. 2017; 13(2):608-27.

12. Fernandes ALC, Sousa VL, Bezerra ALD, Suáres LAB; Mazzaro VDM; Andrade M, Sousa MNA. Qualidade de vida e estresse ocupacional em trabalhadores de presídios. R. Eletr. de Eng. de Produção e Correlatas [Internet]. 2016; 16(1):263-277.

13. Oliveira $A M B$, Fonseca CC, Brandão TS. 0 cotidiano na cadeia pública de Caraubas/RN/Brasil sob o olhar do agente penitenciário. Revista CCCSS [Internet]. 2020; 1(1):1-13.

14. Araújo RM, Oliveira AL. Complexo Penal Estadual Agrícola Mário Negócio: 0 agente penitenciário e a reintegração social do interno sob parâmetros da crise no sistema prosional brasileiro. Diálogo [Internet]. 2018 38(Supl):75-88.

15. Miot HA. Análise de concordância em estudos clínicos e experimentais. J Vasc Braz. [Internet]. 2016; 15(2): 89-92. 